\title{
Effect of vildagliptin, a dipeptidyl peptidase 4 inhibitor, on cardiac hypertrophy induced by chronic beta-adrenergic stimulation in rats
}

Toru Miyoshi ${ }^{*}$, Kazufumi Nakamura ${ }^{2 *}$, Masashi Yoshida ${ }^{3}$, Daiji Miura ${ }^{4}$, Hiroki Oe ${ }^{5}$, Satoshi Akagi ${ }^{2}$, Hiroki Sugiyama $^{2}$, Kaoru Akazawa², Tomoko Yonezawa ${ }^{6}$, Jun Wada $^{7}$ and Hiroshi Ito ${ }^{2}$

\begin{abstract}
Background: Heart failure with left ventricular (LV) hypertrophy is often associated with insulin resistance and inflammation. Recent studies have shown that dipeptidyl peptidase 4 (DPP4) inhibitors improve glucose metabolism and inflammatory status. We therefore evaluated whether vildagliptin, a DPP4 inhibitor, prevents LV hypertrophy and improves diastolic function in isoproterenol-treated rats.

Methods: Male Wistar rats received vehicle $(n=20)$, subcutaneous isoproterenol $(2.4 \mathrm{mg} / \mathrm{kg} /$ day, $n=20)(I S O)$, subcutaneous isoproterenol (2.4 mg/kg/day + oral vildagliptin (30 mg/kg/day, $n=20)$ (ISO-VL), or vehicle + oral vildagliptin (30 mg/kg/day, $n=20$ ) (vehicle-VL) for 7 days.

Results: Blood pressure was similar among the four groups, whereas LV hypertrophy was significantly decreased in the ISO-VL group compared with the ISO group (heart weight/body weight, vehicle: $3.2 \pm 0.40$, ISO: $4.43 \pm 0.39$, ISO-VL: $4.14 \pm 0.29$, vehicle-VL: $3.16 \pm 0.16, p<0.05$ ). Cardiac catheterization revealed that vildagliptin lowered the elevated LV end-diastolic pressure observed in the ISO group, but other parameters regarding LV diastolic function such as the decreased minimum dp/dt were not ameliorated in the ISO-VL group. Histological analysis showed that vildagliptin attenuated the increased cardiomyocyte hypertrophy and perivascular fibrosis, but it did not affect angiogenesis in cardiac tissue. In the ISO-VL group, quantitative PCR showed attenuation of increased mRNA expression of tumor necrosis factor-a, interleukin-6, insulin-like growth factor-I, and restoration of decreased mRNA expression of glucose transporter type 4.
\end{abstract}

Conclusions: Vildagliptin may prevent LV hypertrophy caused by continuous exposure to isoproterenol in rats.

Keywords: Cardiac hypertrophy, Dipeptidyl peptidase 4 inhibitor, Inflammation, Left ventricular diastolic dysfunction

\section{Background}

Heart failure with preserved ejection fraction (HFpEF) is the most common form of heart failure in patients with hypertension. HFpEF is associated with considerable morbidity and mortality, and the risk of adverse outcome increases with the severity of diastolic dysfunction [1]. Treatment with anti-hypertensive drugs may be effective for the prevention of HFpEF; however, anti-

\footnotetext{
* Correspondence: miyoshit@cc.okayama-u.ac.jp; ichibun@cc.okayama-u.ac.jp 'Department of Cardiovascular Therapeutics, Okayama University Graduate School of Medicine, Dentistry and Pharmaceutical Sciences, Okayama, Japan ${ }^{2}$ Department of Cardiovascular Medicine, Okayama University Graduate School of Medicine, Dentistry and Pharmaceutical Sciences, 2-5-1, Shikata-cho, Okayama 700-8558, Japan

Full list of author information is available at the end of the article
}

hypertensive drugs including angiotensin-converting enzyme inhibitor and angiotensin receptor blocker have failed to show a benefit to mortality or morbidity. Interestingly, patients with HFpEF often have systemic insulin resistance [2]. Recent studies have shown that systemic insulin resistance contributes to dysregulated insulin and metabolic signaling in the heart and the development of diastolic dysfunction [3]. Furthermore, insulin resistance is accompanied with chronic low-grade inflammation [4], leading to the pathogenesis of hypertension and chronic heart failure [5,6]. Accordingly, there has been increased interest in restoring energy metabolism and establishing anti-inflammatory therapies for HFpEF. 
Glucagon-like peptide-1 (GLP-1 [7-35]) amide is an incretin hormone secreted mainly by the entero-endocrine cells of the intestine in response to the presence of nutrients [7]. GLP-1 receptors are expressed in rodent and human heart [8] and augment the intake of glucose to myocytes to improve energy metabolism [9]. GLP-1 was also reported to enhance L-type $\mathrm{Ca}^{2+}$ current in isolated cardiomyocytes via activation of the cAMP-dependent protein kinase A mechanism [10]. An experimental study showed that a GLP-1 analog had protective effects on high-fat diet-induced insulin resistance [11], inflammation [12], and myocardial infarction [13]. However, the in vivo half-life of GLP-1 is very short (2 to $3 \mathrm{~min}$ ) because it is degraded by dipeptidyl peptidase 4 (DPP4), and thus DPP4 inhibitors lead to increased GLP-1 levels in the blood and extend the duration of GLP-1 action. Recent experimental studies evaluated the protective effect of DPP4 inhibitors in cardiovascular disease in hypertension [14], heart failure [15], and myocardial infarction [16-18].

Recently, we reported that continuous infusion of isoproterenol (ISO) induces cardiac hypertrophy and diastolic dysfunction in rats [19]. Despite the abovementioned studies on cardiac function, the effects of DPP4 inhibition on cardiac inflammation and cardiac insulin resistance have not been fully elucidated. Therefore, the purpose of this study was to investigate whether a DPP4 inhibitor, vildagliptin, can prevent left ventricular (LV) hypertrophy and LV diastolic function in isoproterenol-treated rats.

\section{Methods}

\section{Animals and drug infusion}

Male Wistar rats weighing 193-222 g (8 weeks old; CLEA Japan, Inc.) were used as described [19]. Delivery of isoproterenol or vehicle was achieved by subcutaneously implanting an osmotic minipump (Alzet, model 2001; $1.0 \mu \mathrm{L} / \mathrm{h}$ ) in the neck under diethyl ether inhalation anesthesia. Rats were divided into four groups and treated for 7 days: vehicle group (subcutaneous $\mathrm{pH} 4.0 \mathrm{HCl}$ in saline, $n=10$ ), ISO group (subcutaneous isoproterenol $2.4 \mathrm{mg} / \mathrm{kg} /$ day, $n=20$ ), isoproterenol + vildagliptin group (ISO-VL; subcutaneous isoproterenol $2.4 \mathrm{mg} / \mathrm{kg} /$ day and oral vildagliptin $30 \mathrm{mg} / \mathrm{kg} /$ day, $n=20$ ), and vehicle + vildagliptin group (subcutaneous $\mathrm{pH} 4.0 \mathrm{HCl}$ in saline and oral vildagliptin $30 \mathrm{mg} / \mathrm{kg} /$ day, $n=20$ ). Vildagliptin was administered by gavage in $0.5 \%$ carboxymethyl cellulose sodium. All animal protocols were approved and conducted according to the recommendations of the Okayama University Animal Care and Use Committee.

\section{Oral glucose tolerance test}

After rats were fasted for $18 \mathrm{~h}$, glucose was orally administered $(2 \mathrm{~g} / \mathrm{kg})$. Blood samples were obtained before and 30, 60, 90, and $120 \mathrm{~min}$ after glucose loading. Blood glucose concentrations were measured immediately with a blood glucose monitor (Accu-Check, Roche), and data were quantified by calculating the area under the curve (AUC 0-120 min) using the trapezoidal rule.

\section{Measurements of plasma active GLP-1, brain natriuretic peptide, and pentosidine}

Active (uncleaved, 7-36 amide or 7-37) GLP-1 was detected using the commercially available enzyme-linked immunosorbent assay kit (Millipore) using mouse insulin standards; each sample was analyzed in duplicate. This antibody only detects active GLP-1 (7-36 amide or 7-37 GLP-1) but not cleaved GLP- 1 (9-36 amide or 9-37 GLP-1). Plasma brain natriuretic peptide and pentosidine were measured using an enzyme-linked immunosorbent assay method at SRL Company Ltd. (Tokyo, Japan).

\section{DPP4 activity measurements}

DPP4 activity was determined by the cleavage rate of 7 amino-4-methylcoumarin (AMC) from the synthetic substrate $H$-glycyl-prolyl-AMC (Gly-Pro-AMC; Sigma). Briefly, $5 \mu \mathrm{l}$ of sample was mixed with $35 \mu \mathrm{l}$ of assay buffer (25 mM HEPES). After 5-min preincubation at room temperature, the reaction was initiated by the addition of $40 \mu \mathrm{l}$ of assay buffer containing $0.1 \mathrm{mM}$ substrate Gly-Pro-AMC. After incubation for $20 \mathrm{~min}$, fluorescence was determined using a spectrofluorometer (excitation $380 \mathrm{~nm} /$ emission $460 \mathrm{~nm}$ ). The standard curve of free AMC was generated using 0-50 mM AMC (Sigma). DPP4 activity in plasma was expressed as the amount of cleaved AMC per minute per $\mathrm{ml}(\mathrm{nmol} / \mathrm{min} / \mathrm{ml})$.

\section{Echocardiography}

Seven days after infusion, transthoracic echocardiography was performed using a $10-\mathrm{MHz}$ phased array transducer (Aplio ver. 6.0, Toshiba, Japan) under 2\% isoflurane. An electrocardiogram was acquired simultaneously. End diastole was defined as the peak of the $\mathrm{R}$ wave, and end systole was defined as the end of the $\mathrm{T}$ wave. All animals underwent echocardiographic interrogation while lying in a left recumbent position. Parasternal short-axis and apical long-axis views were obtained. M-mode echocardiography was performed using a parasternal short-axis view at the level of the papillary muscles. LV posterior and interventricular septal diastolic wall thicknesses (PWT and IVST) were measured during diastole (d) and systole (s) as were the LV internal diameters at end diastole (LVDd) and end systole (LVDs). Fractional shortening (FS) was then calculated according to the formula FS $=[($ LVDd - LVDs $) / L V D d] \times 100$. LV mass $(L V M)$ was calculated according to $1.04 \times[(\mathrm{LVDd}+\mathrm{PWT}+$ IVST $^{3}-(\text { LVDd })^{3}$ ] [20] and LV mass index (LVMI) was calculated by normalizing LVM for body weight. The 
apical four-chamber view was used to assess early and late transmitral peak diastolic flow velocities ( $\mathrm{E}$ and A waves, respectively).

\section{Hemodynamic measurements}

Seven days after infusion, rats were anesthetized, and a micro-tip pressure transducer (Millar Instruments Inc., Houston, TX, USA) was inserted into the right carotid artery. Arterial systolic and diastolic blood pressures were recorded in the aortic arch. The catheter was advanced into the LV cavity. After a 5-min period of stabilization, heart rate, LV systolic pressure (LVSP), LV end-diastolic pressure (LVEDP), and developed LV pressure $(\mathrm{dLVP}=\mathrm{LVSP}$ - LVEDP) were measured. For indices of contractility and relaxation, the maximal rates of increase and decrease in LVP $\mathrm{dp} / \mathrm{dt}$ maximum and $\mathrm{dp} / \mathrm{dt}$ minimum were determined.

\section{Histology}

The LV was fixed with $4 \%$ paraformaldehyde in phosphate buffered saline (PBS), embedded in paraffin, and cut into 5 - $\mu$ m-thick sections. Sections were stained with hematoxylin and eosin for morphological analysis and with Masson's trichrome to detect fibrosis and then examined by light microscopy. In sections stained with Masson's trichrome, interstitial fibrosis was measured using computer-assisted image analysis, and the percent fibrosis was calculated [21,22]. The widths of 30 individual cardiomyocytes in each group were measured as previously described. To visualize the capillaries in the myocardium, endothelial cells in frozen sections were stained with anti-rat CD31 Ab (BD Pharmingen) at 1:100 dilution. The antibody was visualized with a horseradish peroxidase (HRP)-conjugated goat anti-rat IgG and streptavidin-HRP complex (Vectastain ABC elite kit, Vector). HRP activity was detected with $0.025 \%$ diaminobenzidine and $0.03 \% \mathrm{H}_{2} \mathrm{O}_{2}$ in PBS. Images were captured with a light microscope, and capillaries were evaluated as the number of vessels per cardiomyocyte.

\section{Quantitative PCR}

Total RNA was extracted from heart tissue using Trizol (Invitrogen, Carlsbad, CA, USA). Total RNA (2 $\mu \mathrm{g})$ was reverse-transcribed using ReverTra Ace (TOYOBO, Osaka, Japan). Finally, the cDNAs were diluted 5-fold before conventional reverse transcription-PCR (RT-PCR) amplification or 50-fold before quantitative PCR analysis. Quantitative real-time RT-PCR was performed using a LightCycler rapid thermal cycler system (Roche Applied Science) as reported [23]. The PCR primers were as follows (forward primer and reverse primer): tumor necrosis factor-alpha (Tnfa), 5'-TGAACTTCGGGGTGATCG-3' and 5' - GGGCTTGTCACTCGAGTTTT-3'; interleukin6 (Il6), 5'-GCCCTTCAGGAACAGCTATG-3' and 5'-G
CAGTGGCTGTCAACAACA-3'; $\alpha$-myosin heavy chain (Myh6), 5' -CATGCGCATTGAGTTCAAGA-3' and 5'TCATCCACGGCCAATTCT-3'; glucose transporter type 4. (Glut4), 5'-TTGCAGTGCCTGAGTCTTCTT-3' and 5'-CCAGTCACTCGCTGCTGA-3'; insulin-like growth factor-1 (Igf1), 5'-ATGCCCAAGACTCAGAAGGA-3' and 5' -CGTGGCATTTTCTGTTCCTC-3'; dipeptidylpeptidase 4 (Dpp 4), 5'-CTCCAGAGGACAACCTTGAC-3' and 5'-GGACAAGTGTGCTCTTGAGT-3'; glyceraldehyde 3-phosphate dehydrogenase (Gapdh), 5'-GGCAAGTTCA ATGGCACAGT-3' and 5'-TGGTGAAGACGCCAGTAG ACTC-3'. Gapdh served as an internal control. Data were analyzed using the standard curve method.

\section{Statistical analysis}

All data are expressed as the mean \pm SE. The two-tailed Student's $t$-test for two groups and one-way analysis of variance followed by Tukey post-hoc test for more than two groups were used. $p$-values $<0.05$ were considered significant.

\section{Results}

Oral glucose tolerance test and active GLP-1 levels

Oral glucose tolerance and plasma GLP-1 levels in the vehicle, ISO, vehicle-VL, or ISO-VL groups are shown in Figure 1. The glucose levels at 120 minutes after glucose loading were significantly lower in the ISO-VL and vehicle-VL groups than in the vehicle and ISO groups (Figure 1A). AUCs were also significantly smaller in the ISO-VL and vehicle-VL groups than in the vehicle and ISO groups (ISO-VL, $244 \pm 7 \mathrm{mg}$; vehicle-VL $243 \pm$ $6 \mathrm{mg} \mathrm{min} / \mathrm{dl}$; vehicle, $264 \pm 7 \mathrm{mg} \mathrm{min} / \mathrm{dl}$ : ISO, $265 \pm$ $8 \mathrm{mg} \mathrm{min} / \mathrm{dl}, p<0.05)$. Active GLP-1 levels at the fasting state were significantly higher in the ISO-VL and vehicleVL groups than in the vehicle and ISO groups (Figure 1B), and DPP4 activity at the fasting state was significantly lower in the ISO-VL and vehicle-VL groups than in the vehicle and ISO groups (Figure 1C).

\section{Body and heart weight}

Body weight, heart weight, and the heart-to-body weight ratio after 7 days of treatment are shown in Table 1 . We observed no difference in body weight among the four groups at baseline or at 7 days post-treatment with vehicle, ISO, vehicle-VL, or ISO-VL. The increases in heart weight and heart-to-body weight ratio observed for the ISO group were significantly suppressed in the ISO-VL group $(p<0.05)$. Vildagliptin alone did not affect heart weight or heart-to-body weight ratio. Therefore, histological, hemodynamic and gene expression analyses were performed only in rats treated with vehicle, ISO, or ISO-VL. 

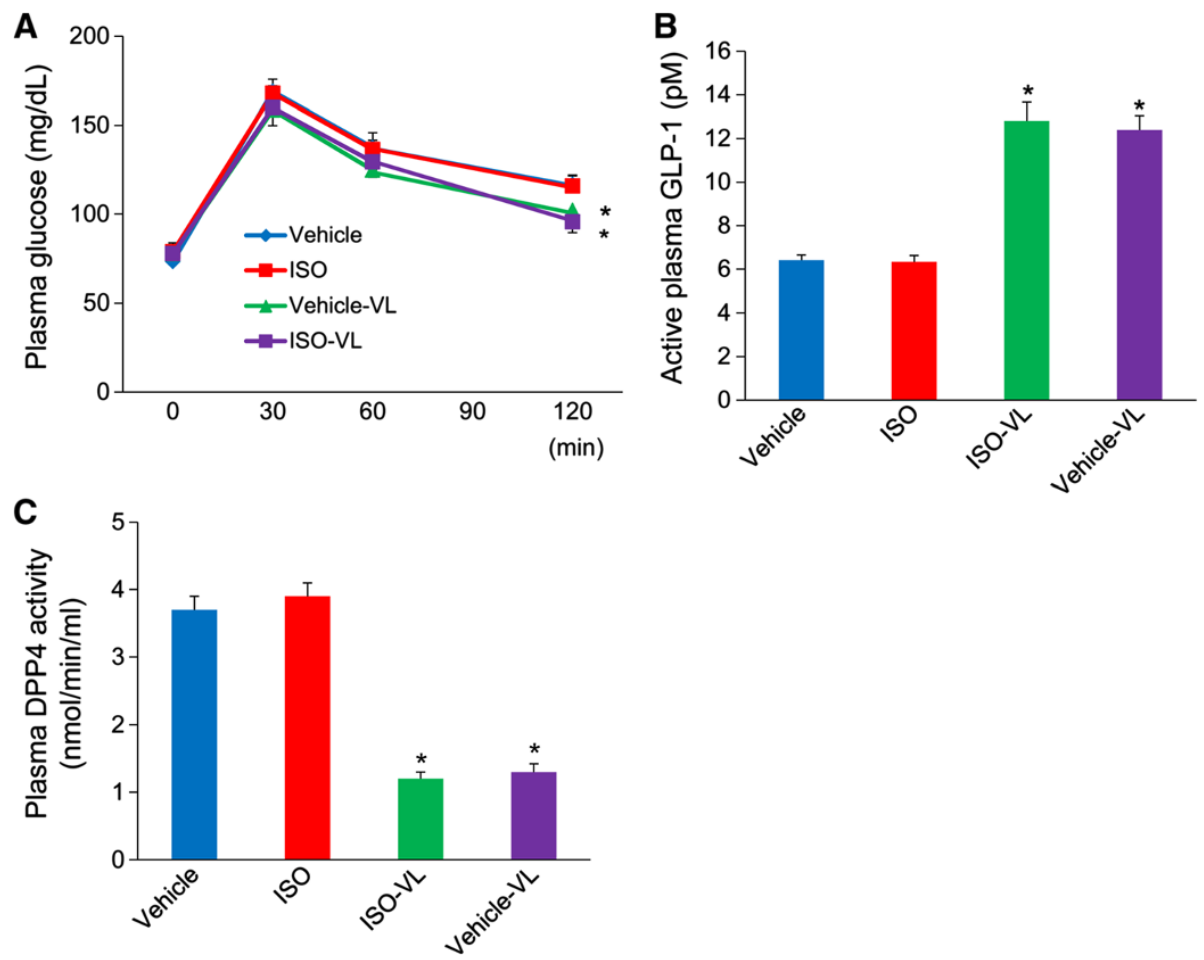

Figure 1 Effects of vildagliptin on glucose tolerance, active GLP-1 levels, and DPP4 activity in isoproterenol-treated rats. Plots of blood glucose over time (A), levels of active plasma glucagon-like peptide (GLP)-1 (B), and dipeptidyl peptidase (DPP)-4 activity (C) in vehicle, ISO, ISO-VL, and vehicle-VL groups. Data represent the mean \pm SE of 10 rats. ${ }^{*} p<0.05$ vs. the vehicle and ISO groups.

\section{Histology}

Hematoxylin and eosin staining after 7 days of treatment revealed that the increase in cardiomyocyte width in the ISO group was significantly suppressed in the ISO-VL group (cardiomyocyte width: vehicle, $10.4 \pm 0.2 \mu \mathrm{m}$; ISO, $13.4 \pm 0.4 \mu \mathrm{m}$; ISO-VL, $11.1 \pm 0.2 \mu \mathrm{m}$; Figure 2). Masson's trichrome staining revealed that the area of perivascular fibrosis was significantly suppressed in the ISO-VL group compared with the ISO group (area of fibrosis: vehicle, $4.1 \pm 1.8 \%$; ISO, $7.4 .5 \pm 1.8 \%$; ISO-VL, $5.8 \pm 1.6 \%$; Figure $3 \mathrm{~A}$ ), but vildagliptin did not limit the increased area of interstitial fibrosis caused by isoproterenol (area of fibrosis: vehicle, $0.72 \pm 0.1 \%$; ISO, $0.98 \pm 0.2 \%$; ISO-VL, $0.90 \pm$ $0.1 \%$; Figure $3 \mathrm{~B})$. CD31 staining revealed that the number of CD31-positive capillaries/cardiomyocyte did not differ among the three groups (vessels/cardiomyocyte: vehicle, $1.2 \pm 0.1 \%$; ISO, $1.1 \pm 0.1 \%$; ISO-VL, $1.2 \pm 0.1 \%$; Figure 4).

\section{Hemodynamic and echocardiographic data}

In non-invasive tail-cuff blood pressure measurements, systolic blood pressure at day 7 was significantly higher in the ISO-VL group than in the vehicle and ISO groups $(127 \pm 6,119 \pm 5$, and $117 \pm 5 \mathrm{mmHg}$, respectively, $\mathrm{p}<0.05$ ); however, cardiac catheterization showed no differences in systolic pressure among the three groups. As shown in Table 2, hemodynamic data by cardiac catheterization revealed diastolic dysfunction in ISOtreated rats. ISO-treated rats showed significantly increased maximum $\mathrm{dp} / \mathrm{dt}$, depressed minimum $\mathrm{dp} / \mathrm{dt}$, and elevated LVEDP compared with vehicle-treated rats, but there was no significant difference in LVSP between the three groups. Vildagliptin significantly improved LVEDP in ISO-treated rats, whereas no significant differences were found in maximum $\mathrm{dp} / \mathrm{dt}$ or minimum $\mathrm{dp} / \mathrm{dt}$ between the ISO group and the ISO-

Table 1 Effects of vildagliptin on heart weight

\begin{tabular}{lcccc}
\hline & Vehicle & ISO & ISO-VL & Vehicle-VL \\
\hline Number of rats & 10 & 20 & 20 & 20 \\
Body weight, g & $237 \pm 7$ & $235 \pm 10$ & $234 \pm 11$ & $237 \pm 6$ \\
Heart weight, g & $0.76 \pm 0.10$ & $1.04 \pm 0.11^{*}$ & $0.97 \pm 0.10^{*} \#$ & $0.75 \pm 0.09$ \\
Heart weight/body weight $\times 10^{3}$ & $3.21 \pm 0.40$ & $4.43 \pm 039^{*}$ & $4.14 \pm 0.29^{*} \#$ & $3.16 \pm 0.16$
\end{tabular}

ISO, isoproterenol; VL, vildagliptin. Values represent the mean \pm SE. ${ }^{*} p<0.05$ vs. vehicle and vehicle-VL; \#p $<0.05$ vs. ISO. 


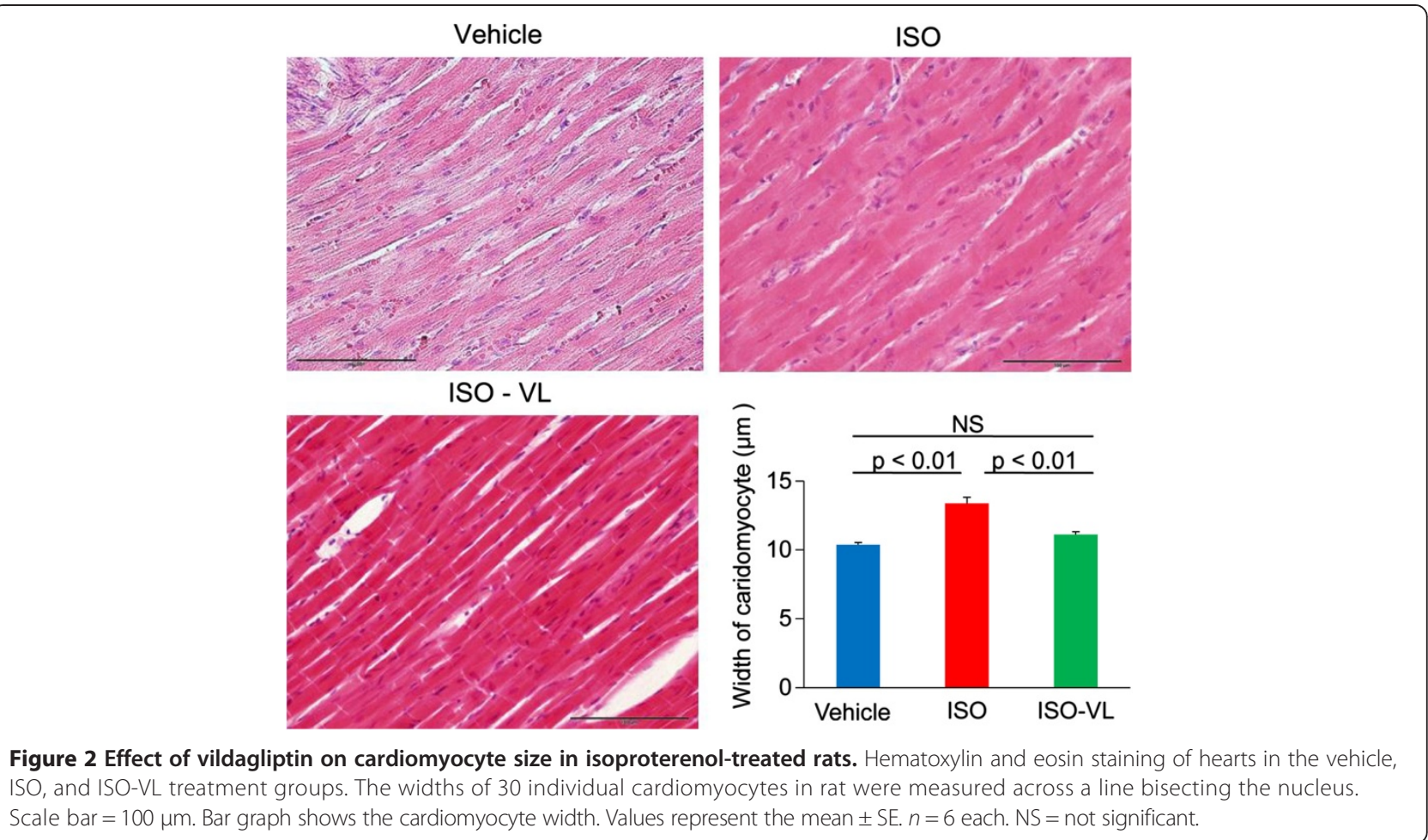

VL group. Echocardiography showed no differences in LV systolic function among the three groups, and vildagliptin attenuated the increase in LVM and LVMI in ISO-treated hearts (Table 2).

\section{Cardiac gene expression}

Figure 5 shows levels of mRNA expression in the heart after treatment. The increased expression of genes that encode inflammatory markers such as Tnfa and Il6 upon ISO treatment was significantly suppressed by vildagliptin (Figure 5A and B). Expression of the gene that encodes Myh6, which is the dominant major histocompatibility complex (MHC) isoform expressed in normal hearts, was significantly decreased in the ISO group (Figure 5C). Vildagliptin significantly limited the decrease in expression of Myh6 mRNA. The mRNA expression of the gene that encodes Glut4, which mediates glucose uptake, was significantly reduced by isoproterenol treatment. Vildagliptin also significantly limited the decrease in Glut4 mRNA (Figure 5D). The increased expression of $I g f 1$ mRNA upon isoproterenol treatment was significantly suppressed by vildagliptin (Figure 5E). The expression of Dpp 4 mRNA was not affected by isoproterenol treatment (Figure 5F).

\section{Measurements of brain natriuretic peptide and pentosidine}

After 7 days of treatment, the increase in brain natriuretic peptide (BNP) level in the ISO group was significantly suppressed in the ISO-VL group (vehicle, $52.2 \pm 2.3 \mathrm{pg} / \mathrm{ml}$; ISO, $74.7 \pm 6.9 \mathrm{pg} / \mathrm{ml}$; ISO-VL, $62.0 \pm 2.3 \mathrm{pg} / \mathrm{ml}, p<0.05)$.
There was no difference in the plasma levels of pentosidine, which is an advanced glycation end product and is generated by non-enzymatic glycation and oxidation of proteins (vehicle, $9.4 \pm 0.51 \mu \mathrm{g} / \mathrm{ml}$; ISO, $8.8 \pm 0.63 \mu \mathrm{g} / \mathrm{ml}$; ISO-VL, $9.4 \pm 0.52 \mu \mathrm{g} / \mathrm{ml})$.

\section{Discussion}

We demonstrated that short-term treatment with a DPP4 inhibitor, vildagliptin, prevented LV hypertrophy caused by continuous infusion of isoproterenol. These effects were accompanied by the amelioration of expression of genes associated with glucose uptake and inflammation. Although cardiac catheterization showed that vildagliptin did not significantly improve LV diastolic dysfunction in isoproterenol-treated rats, this study indicated that vildagliptin has potential for preventing LV hypertrophy independent of blood pressure.

Regarding the mechanisms underlying the protective effect of vildagliptin in preventing isoproterenol-induced LV hypertrophy in this study, one possible explanation is that vildagliptin may reduce inflammation in the heart. A previous study showed that isoproterenol induces expression of mRNAs that encode myocardial pro-inflammatory cytokines such as TNF- $\alpha$, IL-6, and IL-1 $\beta$ [24]. Our previous study revealed a direct effect of TNF- $\alpha$ on cardiac hypertrophy in cultured cardiomyocytes [25]. Thus, TNF- $\alpha$ and IL- 6 are likely to be important factors in the induction of hypertrophy [26,27]. In our current study, vildagliptin reduced the expression of Tnfa and Il6 mRNAs in 

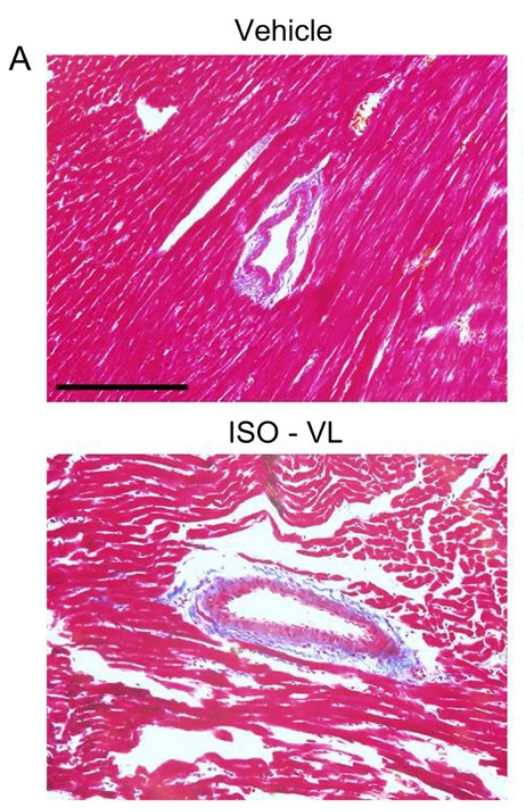

Vehicle

B

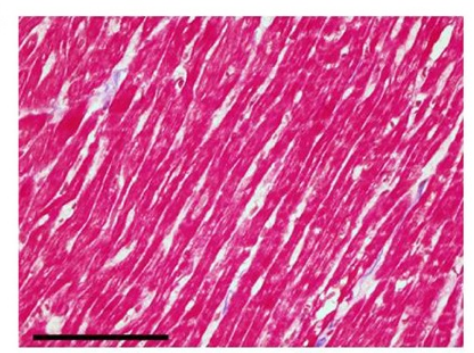

ISO - VL

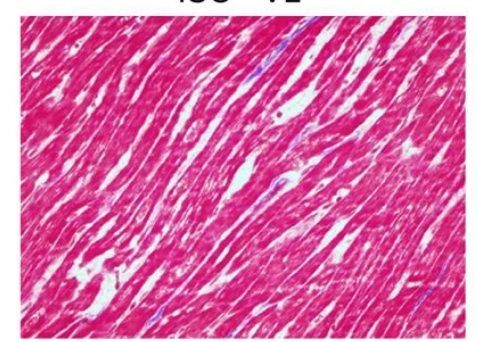

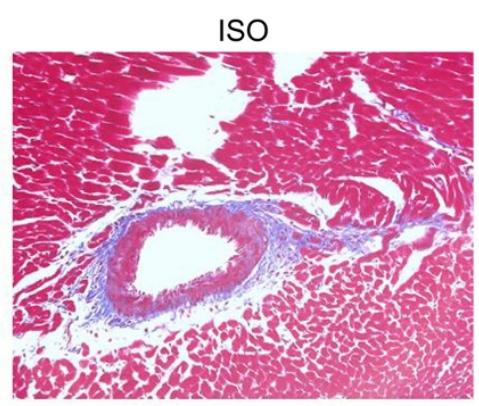

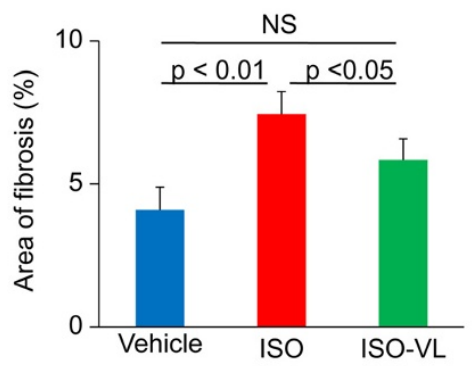

ISO
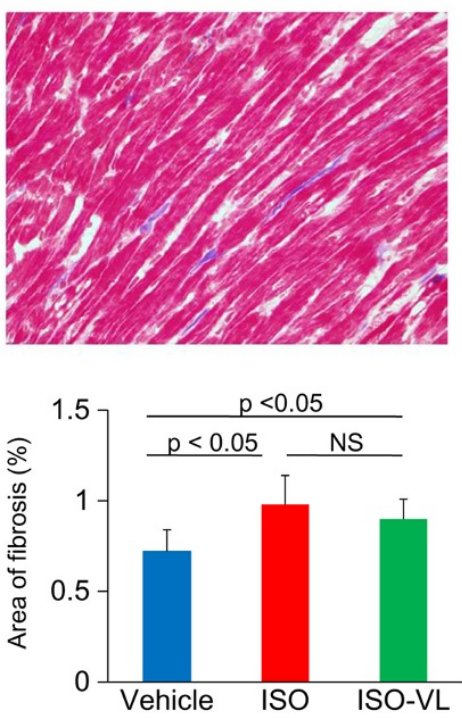

Figure 3 Effect of vildagliptin on cardiac fibrosis in isoproterenol-treated rats. Masson's trichrome staining of hearts in the vehicle, ISO, and ISO-VL treatment groups. A: Perivascular fibrosis. B: Interstitial fibrosis. Scale bars $=100 \mu \mathrm{m}$. Bar graphs show the area of fibrosis (\%) in each case. Values represent the mean \pm SE. $n=6$ each. NS, not significant.

myocardial tissue of isoproterenol-treated rats. Similarly, recent studies demonstrated the effect of DPP4 inhibitors on the reduction of pro-inflammatory cytokines in macrophages, visceral adipose tissue, and atherosclerotic plaques $[28,29]$. Furthermore, vildagliptin suppressed the increase in Igf1 expression induced by isoproterenol in our rat model. Recent studies showed the involvement of IGF1 in cardiomyocyte hypertrophy [30,31]. Thus, a change in cytokine expression by vildagliptin may contribute to the prevention of LV hypertrophy. Another possibility is that increased active GLP-1 levels by vildagliptin directly influences LV hypertrophy. In several previous experiments in rats, oral vildagliptin was used at a dose of 3 to $60 \mathrm{mg} / \mathrm{kg} /$ day [11,32]. We selected the dose of $30 \mathrm{mg} / \mathrm{kg} /$ day of vildagliptin in this study and confirmed that this dosage increased the GLP-1 level at the fasting state by 2 -fold compared with the control group. A study showed that recombinant GLP-1 infusion for 14 days reduces blood pressure, LV hypertrophy, and LV fibrosis in Dahl saltsensitive rats [33]. Another group has reported that administration of a GLP-1 analog diminishes cardiac hypertrophy and blood pressure in obese mice exhibiting 


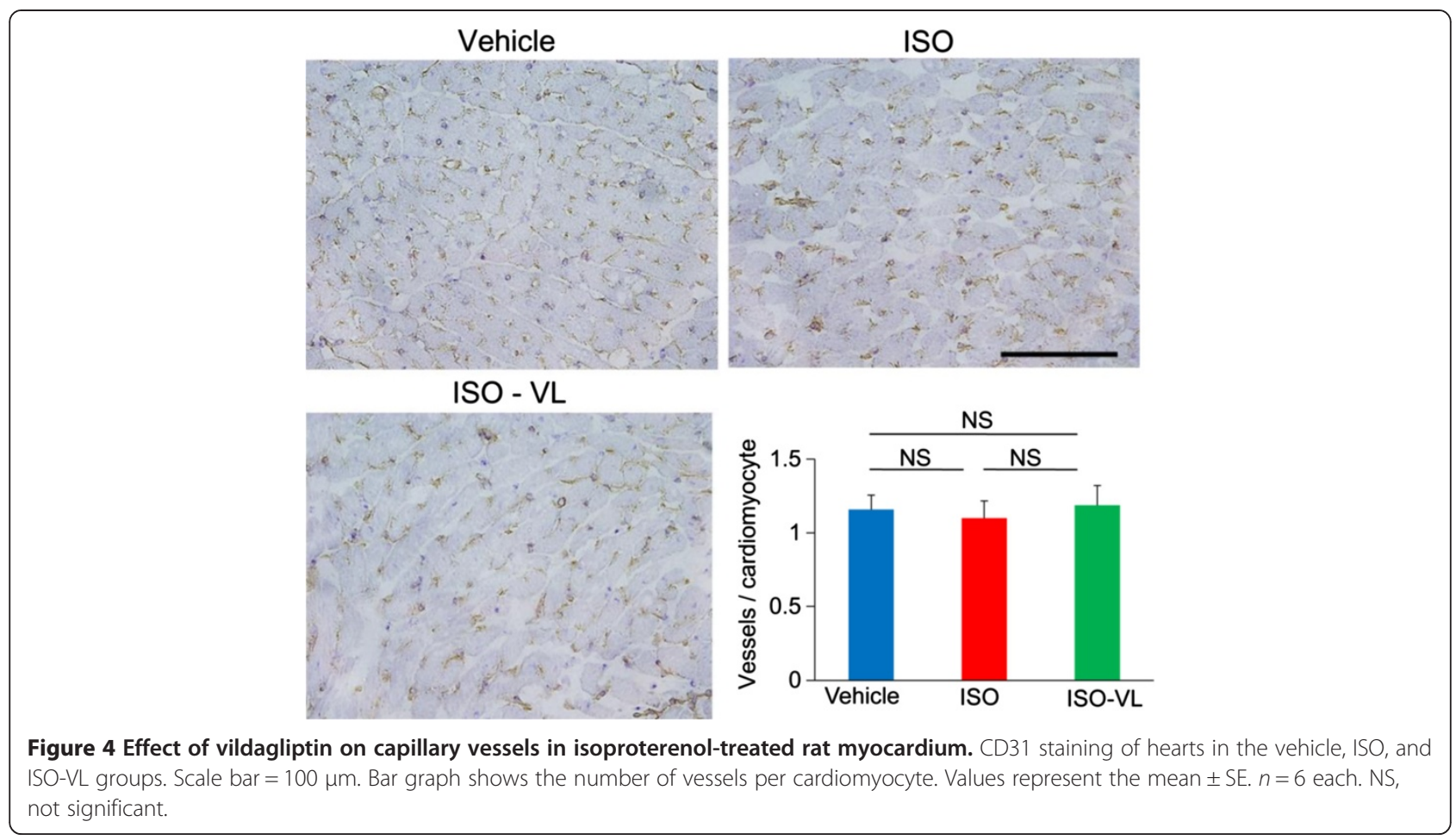

Table 2 Effects of vildagliptin on LV hemodynamic and echocardiographic data

\begin{tabular}{lccc}
\hline & Vehicle & ISO & ISO-VL \\
\hline Cardiac catheterization & 10 & 10 & 10 \\
Number of rats & $404 \pm 8$ & $519 \pm 7^{*}$ & $491 \pm 8^{*} \#$ \\
Heart rate (bpm) & $108 \pm 3$ & $113 \pm 2$ & $116 \pm 2$ \\
LVSP (mmHg) & $2.2 \pm 1.1$ & $3.4 \pm 0.9^{*}$ & $2.5 \pm 1.0 \#$ \\
LVEDP (mmHg) & $8992 \pm 434$ & $16,969 \pm 592^{*}$ & $16,189 \pm 713^{*}$ \\
Maximum dp/dt (mmHg/s) & $-8251 \pm 531$ & $-9936 \pm 459^{*}$ & $-10080 \pm 236^{*}$ \\
Minimum dp/dt (mmHg/s) & & & 10 \\
Echocardiography & 10 & $6.71 \pm 0.04$ & 10 \\
Number of rats & $6.78 \pm 0.14$ & $3.29 \pm 0.08$ & $6.74 \pm 0.08$ \\
LVDd (mm) & $3.62 \pm 0.14$ & $51.0 \pm 1.1$ & $3.41 \pm 0.07$ \\
LVDs (mm) & $46.7 \pm 2.7$ & $1.57 \pm 0.23^{*}$ & $50.0 \pm 0.9$ \\
FS (\%) & $1.34 \pm 0.04$ & $1.62 \pm 0.03^{*}$ & $1.51 \pm 0.03^{*}$ \\
IVST (mm) & $1.38 \pm 0.04$ & $1.57 \pm 0.07$ & $1.56 \pm 0.03^{*}$ \\
PWT (mm) & $1.61 \pm 0.24$ & $695 \pm 9^{*}$ & $1.53 \pm 0.05$ \\
E/A & $567 \pm 16$ & $2.96 \pm 0.04^{*}$ & $663 \pm 11^{*} \#$ \\
LVM (mg) & $2.36 \pm 0.06$ & $2.83 \pm 0.04^{*} \#$ \\
LVMI (mg/g) & &
\end{tabular}

ISO, isoproterenol; VL, vildagliptin; LVSP, left ventricular systolic pressure; LVEDP, left ventricular end diastolic pressure; LVDd, left ventricular end-diastolic diameter; LVDs, left ventricular end-systolic diameter; FS, fractional shortening; IVST, interventricular septal thickness; PWT, posterior wall thickness; E, early transmitral peak diastolic flow velocity; A, late transmitral peak diastolic flow velocity; LVM, left ventricular mass; LVMI, left ventricular mass index. Values represent the mean \pm SE. ${ }^{*} p<0.05$ vs. vehicle; $\# p<0.05$ vs. ISO. 
A
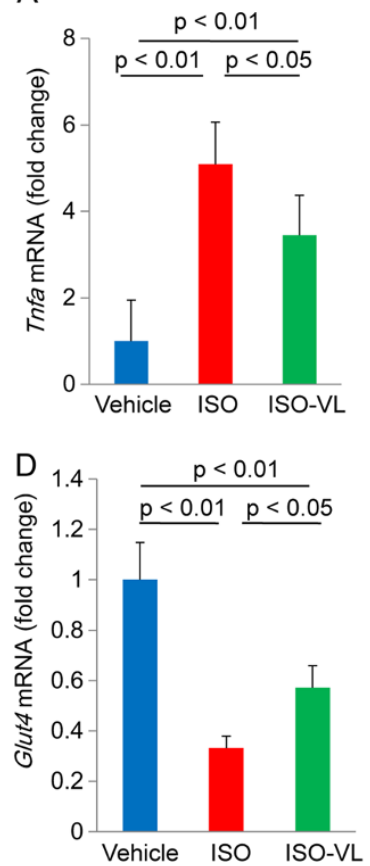

B

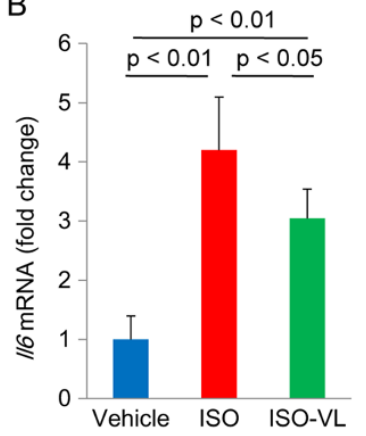

$\mathrm{E}$

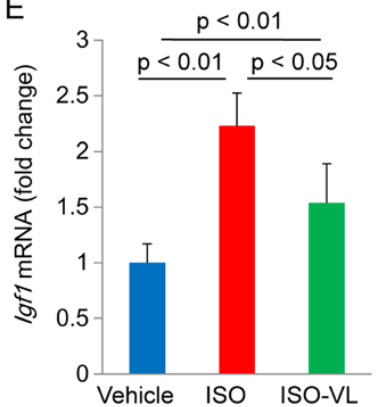

C

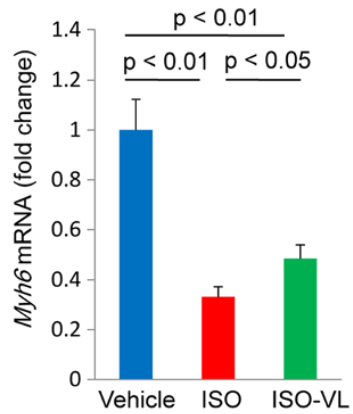

$\mathrm{F}$

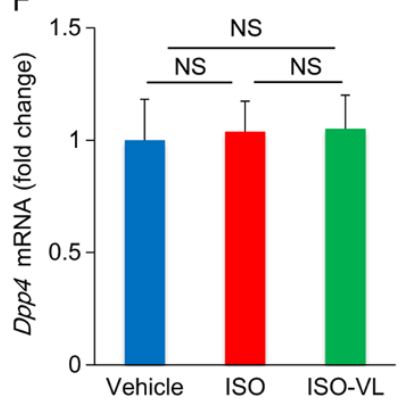

Figure 5 Cardiac gene expression in isoproterenol-treated rats. The changes in expression of tumor necrosis factor alpha (Tnfa) (A), interleukin-6 (II6) (B), a-myosin heavy chain (Myh6) (C), glucose transporter type 4 (Glut4) (D), insulin-like growth factor (Igfi) (E), and dipeptidyl peptidase-4 (Dpp4) (F) mRNAs were analyzed using quantitative PCR. Values represent the mean \pm SE. $n=8$ each. NS, not significant.

insulin resistance [34]. In both studies, it was difficult to discriminate the effect of GLP-1 on the protection of LV hypertrophy from its blood pressure-lowering effects. Taken together, these results suggest that the anti-inflammatory effect and suppression of IGF1 by vildagliptin in the heart at least partly counters LV hypertrophy.

In this study, although LVEDP was significantly lower in the ISO-VL group than in the ISO group, other catheterrelated parameters such as maximum $\mathrm{dp} / \mathrm{dt}$, minimum $\mathrm{dp} / \mathrm{dt}$ were similar between the two groups. Thus, this study failed to demonstrate that vildagliptin ameliorated LV diastolic function in the ISO-VL group. Energy metabolism, however, switches from fatty acid oxidation to carbohydrate oxidation in hypertrophied hearts [35], and thus the increase in expression of Glut4 mRNA by vildagliptin may improve glucose uptake in cardiomyocytes and then ameliorate ATP synthesis through carbohydrate oxidation. In fact, treatment with a DPP4 inhibitor, sitagliptin, improves insulin resistance and increases cardiac GLUT4 protein and mRNA abundance in spontaneously hypertensive rats [36]. In hypertrophied hearts, the shift in $\mathrm{MHC}$ isoform composition from $\alpha$ - to $\beta$-MHC has also been reported [37]. Our finding that expression of cardiac $\alpha-\mathrm{MHC}$ (Myh6) mRNA decreased in isoproterenol-treated rats is consistent with those previous data. The improvements in energy production have beneficial effects on failing hearts and may upregulate Myh6 expression. In this study, although insulin sensitivity was not assessed, rats in the ISO and vehicle groups exhibited similar glucose tolerance patterns. Given that vildagliptin lowered the AUCs in both isoproterenol-treated and vehicle-treated rats, vildagliptin may exert favorable effects on insulin signaling in isoproterenol-treated hearts. This study also demonstrated that decreased inflammatory cytokines may contribute to dysregulated cardiac signaling. Other studies have shown that TNF- $\alpha$ causes cardiac insulin resistance by inducing degradation of insulin receptor substrate protein 1 , which is critical for cardiac insulin signaling $[38,39]$. Thus, reduced expression of Tnfa mRNA by vildagliptin, as shown in our present study, also partly contributes to the increase in energy production in isoproterenol-treated hearts. On the other hand, histological analyses in this study showed that vildagliptin significantly suppressed perivascular fibrosis in isoproterenol-treated hearts but did not affect angiogenesis. One study showed that vildagliptin reverses angiogenesis in diabetic murine hearts by increasing the activity of stromal cell-derived factor- $1 \alpha$, which is a substrate of DPP4 [40]. The lack of change in angiogenesis in the isoproterenol-infused rat model may explain why diastolic function is not significantly improved.

DPP4 is widely expressed on the surface of endothelial cells and immune cells such as lymphocytes and monocytes [40-42]. DPP4 inhibitors exert their effects by inhibiting enzymatic degradation of GLP-1; however, recent studies reported non-enzymatic functions for DPP4 [43]. 
For instance, cell-surface DPP4 regulates inflammatory responses in innate immune cells such as monocytes and dendritic cells through the incretin-independent pathway $[44,45]$. In endothelial cells, DPP4-mediated signaling pathways result in phosphorylation of endothelial nitric oxide synthase [41]. The distinct role of DPP4 in cardiac tissue remains unknown, but further examination will shed light on the novel functions of DPP4 inhibitors in the heart.

Although the effects of DPP4 inhibitors on LV hypertrophy in humans have not been fully elucidated, the moderate blood pressure-lowering effect of DPP4 inhibitor may have favorable effect on LV hypertrophy in this clinical setting [46]. The mechanism of this effect has been attributed to increased diuresis and natriuresis owing to inhibition of sodium reabsorption from the proximal renal tubules [47]. Another mechanism for the blood pressure-lowering effects of DPP4 inhibitors is peripheral vasodilatation and decreased peripheral vascular resistance. Recently, van Poppel et al. showed that vildagliptin improves endothelial function in patients with type 2 diabetes [48]. Moreover, a recent clinical study indicated that endothelial dysfunction is closely associated with HFpEF [49], and thus favorable effects of DPP4 inhibitors on the vascular system suggest a therapeutic potential for preventing LV hypertrophy and HFpEF in humans.

\section{Limitations}

The elevation of the circulating GLP-1 level has a potential role in cardioprotection, but we did not explore the direct effect of GLP-1 on hypertrophy of cardiomyocytes in this study. Further, a wide range of peptides are considered to be substrates of DPP4. Comprehensive analysis of the effect of DPP4 inhibitors on the substrates in hypertrophied hearts will help to identify the underlying mechanisms. Second, our finding indicated the potential role of vildagliptin on the protection, but not regression, of LV hypertrophy. In clinical practice, it would be of interest to see if administration of DPP4 inhibitor has benefits for patients with existing LV hypertrophy. Further study is warranted to address this question. Finally, It is necessary to understand the role of DPP4 itself in cardiac hypertrophy to address the potential role of DPP4 inhibitors as therapeutics.

In conclusion, a DPP4 inhibitor, vildagliptin, prevented LV hypertrophy caused by continuous exposure to isoproterenol in rats. This finding suggests the possibility of using a DPP4 inhibitor to prevent LV hypertrophy in humans.

\footnotetext{
Abbreviations

A: Late transmitral peak diastolic flow velocity; DPP4: Dipeptidyl peptidase 4; E: Early transmitral peak diastolic flow velocity; FS: Fractional shortening; GAPDH: Glyceraldehyde 3-phosphate dehydrogenase; GLP-1: Glucagon-like peptide-1; GLUT4: Glucose transporter type 4; HFpEF: Heart failure with preserved ejection fraction; IGF: Insulin-like growth factor-l; IL6: Interleukin-6; ISO: Isoproterenol; LVMI: Left ventricular mass index; LVDs: Left ventricular internal diameters at end systole; LVEDP: Left ventricular end diastolic pressure; LVSP: Left ventricular systolic pressure; IVST: Interventricular septal diastolic wall
}

thicknesses; IVST: Interventricular septal diastolic wall thicknesses; LVDs: Left ventricular internal diameters at end systole; MYH6: a-myosin heavy chain; PWT: Posterior wall thickness; TNFA: Tumor necrosis factor-alpha; VL: Vildagliptin.

\section{Competing interests}

$\mathrm{JW}$ is a consultant for Boehringer Ingelheim, receives speaker honoraria from Novartis.

\section{Authors' contributions}

TM and KN conceived the study, participated in its design and coordination, and drafted the manuscript. MY, DM, HO, SA, HS, KA, and TY made substantial contributions to acquisition of data or analysis. JW and HI were involved in drafting the manuscript or revising it critically for important intellectual content. All authors' read and approved the final manuscript.

\section{Acknowledgments}

This work was supported in part by a Grant-in-Aid for Scientific Research No. 70444651 (TM), No. 10335630 (KN), No. 23790855 (MY), and No. 50322227 (HM) from the Ministry of Education, Culture, Sports, Science, and Technology, Japan. This study was funded by Novartis Pharma AG.

\section{Author details}

'Department of Cardiovascular Therapeutics, Okayama University Graduate School of Medicine, Dentistry and Pharmaceutical Sciences, Okayama, Japan. ${ }^{2}$ Department of Cardiovascular Medicine, Okayama University Graduate School of Medicine, Dentistry and Pharmaceutical Sciences, 2-5-1,

Shikata-cho, Okayama 700-8558, Japan. ${ }^{3}$ Department of Chronic Kidney Disease and Cardiovascular Disease, Okayama University Graduate School of Medicine, Dentistry and Pharmaceutical Sciences, Okayama, Japan. ${ }^{4}$ Division of Basic and Clinical Medicine, agano College of Nursing, Nagano, Japan. ${ }^{5}$ Center of Ultrasonic Diagnostics, Okayama University Hospital, Okayama, Japan. ${ }^{6}$ Department of Molecular Biology and Biochemistry, Okayama University Graduate School of Medicine, Dentistry and Pharmaceutical Sciences, Okayama, Japan. ${ }^{7}$ Department of Medicine and Clinical Science, Okayama University Graduate School of Medicine, Dentistry and

Pharmaceutical Sciences, Okayama, Japan.

Received: 3 February 2014 Accepted: 6 February 2014

Published: 13 February 2014

\section{References}

1. Bursi F, Weston SA, Redfield MM, Jacobsen SJ, Pakhomov S, Nkomo VT, Meverden RA, Roger VL: Systolic and diastolic heart failure in the community. JAMA 2006, 296(18):2209-2216.

2. Aroor $\mathrm{AR}$, Mandavia $\mathrm{CH}$, Sowers JR: Insulin resistance and heart failure: molecular mechanisms. Heart Fail Clin 2012, 8(4):609-617.

3. Mandavia CH, Aroor AR, Demarco VG, Sowers JR: Molecular and metabolic mechanisms of cardiac dysfunction in diabetes. Life Sci 2013, 92(11):601-608.

4. Calle MC, Fernandez ML: Inflammation and type 2 diabetes. Diabetes Metab 2012, 38(3):183-191.

5. Aukrust P, Ueland T, Muller F, Andreassen AK, Nordoy I, Aas H, Kjekshus J, Simonsen S, Froland SS, Gullestad L: Elevated circulating levels of C-C chemokines in patients with congestive heart failure. Circulation 1998, 97(12):1136-1143.

6. Kobayashi M, Nakamura K, Kusano KF, Nakamura Y, Ohta-Ogo K, Nagase S, Sakuragi S, Ohe T: Expression of monocyte chemoattractant protein-1 in idiopathic dilated cardiomyopathy. Int J Cardiol 2008, 126(3):427-429.

7. Nauck MA, Kleine N, Orskov C, Holst JJ, Willms B, Creutzfeldt W: Normalization of fasting hyperglycaemia by exogenous glucagon-like peptide 1 (7-36 amide) in type 2 (non-insulin-dependent) diabetic patients. Diabetologia 1993, 36(8):741-744.

8. Ban K, Noyan-Ashraf MH, Hoefer J, Bolz SS, Drucker DJ, Husain M: Cardioprotective and vasodilatory actions of glucagon-like peptide 1 receptor are mediated through both glucagon-like peptide 1 receptor-dependent and -independent pathways. Circulation 2008, 117(18):2340-2350.

9. Nikolaidis LA, Elahi D, Hentosz T, Doverspike A, Huerbin R, Zourelias L, Stolarski C, Shen YT, Shannon RP: Recombinant glucagon-like peptide-1 increases myocardial glucose uptake and improves left ventricular performance in conscious dogs with pacing-induced dilated cardiomyopathy. Circulation 2004, 110(8):955-961. 
10. Xiao YF, Nikolskaya A, Jaye DA, Sigg DC: Glucagon-like peptide-1 enhances cardiac L-type Ca2+ currents via activation of the CAMP-dependent protein kinase A pathway. Cardiovasc Diabetol 2011, 10:6.

11. Apaijai N, Pintana $\mathrm{H}$, Chattipakorn SC, Chattipakorn N: Cardioprotective effects of metformin and vildagliptin in adult rats with insulin resistance induced by a high-fat diet. Endocrinology 2012, 153(8):3878-3885.

12. Arakawa M, Mita T, Azuma K, Ebato C, Goto H, Nomiyama T, Fujitani Y, Hirose T, Kawamori R, Watada H: Inhibition of monocyte adhesion to endothelial cells and attenuation of atherosclerotic lesion by a glucagon-like peptide-1 receptor agonist, exendin-4. Diabetes 2010, 59(4):1030-1037.

13. Barakat GM, Nuwayri-Salti N, Kadi LN, Bitar KM, Al-Jaroudi WA, Bikhazi AB: Role of glucagon-like peptide-1 and its agonists on early prevention of cardiac remodeling in type 1 diabetic rat hearts. Gen Physiol Biophys 2011, 30(1):34-44

14. Pacheco BP, Crajoinas RO, Couto GK, Davel AP, Lessa LM, Rossoni LV, Girardi AC: Dipeptidyl peptidase IV inhibition attenuates blood pressure rising in young spontaneously hypertensive rats. J Hypertens 2011, 29(3):520-528.

15. Chaykovska L, von Websky K, Rahnenfuhrer J, Alter M, Heiden S, Fuchs H, Runge $F$, Klein T, Hocher B: Effects of DPP-4 inhibitors on the heart in a rat model of uremic cardiomyopathy. PLoS One 2011, 6(11):e27861.

16. Miki T, Itoh T, Sunaga D, Miura T: Effects of diabetes on myocardial infarct size and cardioprotection by preconditioning and postconditioning. Cardiovasc Diabetol 2012, 11:67

17. Ye Y, Keyes KT, Zhang C, Perez-Polo JR, Lin Y, Birnbaum Y: The myocardial infarct size-limiting effect of sitagliptin is PKA-dependent, whereas the protective effect of pioglitazone is partially dependent on PKA. Am J Physiol Heart Circ Physiol 2010, 298(5):H1454-1465.

18. Yin M, Sillje HH, Meissner M, van Gilst WH, de Boer RA: Early and late effects of the DPP-4 inhibitor vildagliptin in a rat model of postmyocardial infarction heart failure. Cardiovasc Diabetol 2011, 10:85.

19. Sumita Yoshikawa W, Nakamura K, Miura D, Shimizu J, Hashimoto K, Kataoka N, Toyota H, Okuyama H, Miyoshi T, Morita H, Fukushima Kusano K, Matsuo T, Takaki M, Kajiya F, Yagi N, Ohe T, Ito H: Increased Passive Stiffness of Cardiomyocytes in the Transverse Direction and Residual Actin and Myosin Cross-Bridge Formation in Hypertrophied Rat Hearts Induced by Chronic beta-Adrenergic Stimulation. Circ J 2012, 77(3):741-748.

20. Devereux RB, Reichek N: Echocardiographic determination of left ventricular mass in man. Anatomic validation of the method. Circulation 1977, 55(4):613-618.

21. Gallego B, Arevalo MA, Flores O, Lopez-Novoa JM, Perez-Barriocanal F: Renal fibrosis in diabetic and aortic-constricted hypertensive rats. Am J Physiol Regul Integr Comp Physiol 2001, 280(6):R1823-1829.

22. Takemoto M, Egashira K, Tomita H, Usui M, Okamoto H, Kitabatake A, Shimokawa H, Sueishi K, Takeshita A: Chronic angiotensin-converting enzyme inhibition and angiotensin II type 1 receptor blockade: effects on cardiovascular remodeling in rats induced by the long-term blockade of nitric oxide synthesis. Hypertension 1997, 30(6):1621-1627.

23. Yonezawa T, Hattori S, Inagaki J, Kurosaki M, Takigawa T, Hirohata S, Miyoshi T, Ninomiya Y: Type IV collagen induces expression of thrombospondin-1 that is mediated by integrin alpha1beta1 in astrocytes. Glia 2010, 58(7):755-767.

24. Murray DR, Prabhu SD, Chandrasekar B: Chronic beta-adrenergic stimulation induces myocardial proinflammatory cytokine expression. Circulation 2000, 101(20):2338-2341.

25. Nakamura K, Fushimi K, Kouchi H, Mihara K, Miyazaki M, Ohe T, Namba M: Inhibitory effects of antioxidants on neonatal rat cardiac myocyte hypertrophy induced by tumor necrosis factor-alpha and angiotensin II. Circulation 1998, 98(8):794-799.

26. Sekiguchi K, Li X, Coker M, Flesch M, Barger PM, Sivasubramanian N, Mann DL: Cross-regulation between the renin-angiotensin system and inflammatory mediators in cardiac hypertrophy and failure. Cardiovasc Res 2004, 63(3):433-442.

27. Yokoyama T, Nakano M, Bednarczyk JL, McIntyre BW, Entman M, Mann DL: Tumor necrosis factor-alpha provokes a hypertrophic growth response in adult cardiac myocytes. Circulation 1997, 95(5):1247-1252.

28. Vittone F, Liberman A, Vasic D, Ostertag R, Esser M, Walcher D, Ludwig A, Marx N, Burgmaier M: Sitagliptin reduces plaque macrophage content and stabilises arteriosclerotic lesions in Apoe (-/-) mice. Diabetologia 2012, 55(8):2267-2275.

29. Zhong J, Rao X, Rajagopalan S: An emerging role of dipeptidyl peptidase 4 (DPP4) beyond glucose control: potential implications in cardiovascular disease. Atherosclerosis 2013, 226(2):305-314

30. Carrasco L, Cea P, Rocco P, Pena-Oyarzun D, Rivera-Mejias P, Sotomayor-Flores C Quiroga C, Criollo A, Ibarra C, Chiong M, Lavandero S: Role of Heterotrimeric G Protein and Calcium in Cardiomyocyte Hypertrophy Induced by IGF-1. J Cell Biochem 2014, 115(4):712-720.
31. Honsho S, Nishikawa S, Amano K, Zen K, Adachi Y, Kishita E, Matsui A, Katsume A, Yamaguchi S, Nishikawa K, Isoda K, Riches DW, Matoba S, Okigaki M, Matsubara H Pressure-mediated hypertrophy and mechanical stretch induces IL-1 release and subsequent IGF-1 generation to maintain compensative hypertrophy by affecting Akt and JNK pathways. Circ Res 2009, 105(11):1149-1158.

32. Duttaroy A, Voelker F, Merriam K, Zhang X, Ren X, Subramanian K, Hughes TE, Burkey BF: The DPP-4 inhibitor vildagliptin increases pancreatic beta cell mass in neonatal rats. Eur J Pharmacol 2011, 650(2-3):703-707.

33. Yu M, Moreno C, Hoagland KM, Dahly A, Ditter K, Mistry M, Roman RJ: Antihypertensive effect of glucagon-like peptide 1 in Dahl salt-sensitive rats. J Hypertens 2003, 21(6):1125-1135.

34. Mells JE, Fu PP, Sharma S, Olson D, Cheng L, Handy JA, Saxena NK, Sorescu D, Anania FA: Glp-1 analog, liraglutide, ameliorates hepatic steatosis and cardiac hypertrophy in C57BL/6 J mice fed a Western diet. Am J Physiol Gastrointest Liver Physiol 2012, 302(2):G225-235.

35. de las Fuentes L, Herrero P, Peterson LR, Kelly DP, Gropler RJ, Davila-Roman VG: Myocardial fatty acid metabolism: independent predictor of left ventricular mass in hypertensive heart disease. Hypertension 2003, 41(1):83-87.

36. Giannocco G, Oliveira KC, Crajoinas RO, Venturini G, Salles TA, FonsecaAlaniz MH, Maciel RM, Girardi AC: Dipeptidyl peptidase IV inhibition upregulates GLUT4 translocation and expression in heart and skeletal muscle of spontaneously hypertensive rats. Eur J Pharmacol 2013, 698(1-3):74-86.

37. Lowes BD, Minobe W, Abraham WT, Rizeq MN, Bohlmeyer TJ, Quaife RA, Roden RL, Dutcher DL, Robertson AD, Voelkel NF, Badesch DB, Groves BM, Gilbert EM, Bristow MR: Changes in gene expression in the intact human heart. Downregulation of alpha-myosin heavy chain in hypertrophied, failing ventricular myocardium. J Clin Invest 1997, 100(9):2315-2324.

38. Korelitz Bl, Sommers SC: Responses to drug therapy in ulcerative colitis. Evaluation by rectal biopsy and histopathological changes. Am J Gastroenterol 1975, 64(5):365-370

39. Hitomi H, Mehta PK, Taniyama Y, Lassegue B, Seidel-Rogol B, San Martin A, Griendling KK: Vascular smooth muscle insulin resistance, but not hypertrophic signaling, is independent of angiotensin II-induced IRS-1 phosphorylation by JNK. Am J Physiol Cell Physiol 2011, 301(6):C1415-1422.

40. Shigeta T, Aoyama M, Bando YK, Monji A, Mitsui T, Takatsu M, Cheng XW Okumura T, Hirashiki A, Nagata K, Murohara T: Dipeptidyl Peptidase-4 Modulates Left Ventricular Dysfunction in Chronic Heart Failure via AngiogenesisDependent and -Independent Actions. Circulation 2012, 126(15):1838-1851.

41. Deacon CF, Plamboeck A, Rosenkilde MM, de Heer J, Holst JJ: GIP-(3-42) does not antagonize insulinotropic effects of GIP at physiological concentrations. Am J Physiol Endocrinol Metab 2006, 291(3):E468-475.

42. Yazbeck R, Howarth GS, Abbott CA: Dipeptidyl peptidase inhibitors, an emerging drug class for inflammatory disease? Trends Pharmacol Sci 2009, 30(11):600-607.

43. Zhong J, Rao X, Rajagopalan S: An emerging role of dipeptidyl peptidase 4 (DPP4) beyond glucose control: Potential implications in cardiovascular disease. Atherosclerosis 2012, 226(2):305-314.

44. Kim SJ, Nian C, Doudet DJ, McIntosh CH: Dipeptidyl peptidase IV inhibition with MK0431 improves islet graft survival in diabetic NOD mice partially via T-cell modulation. Diabetes 2009, 58(3):641-651.

45. Ta NN, Li Y, Schuyler CA, Lopes-Virella MF, Huang Y: DPP-4 (CD26) inhibitor alogliptin inhibits TLR4-mediated ERK activation and ERK-dependent MMP-1 expression by U937 histiocytes. Atherosclerosis 2010, 213(2):429-435.

46. Chrysant SG, Chrysant GS: Clinical implications of cardiovascular preventing pleiotropic effects of dipeptidyl peptidase-4 inhibitors. Am J Cardiol 2012, 109(11):1681-1685.

47. Moreno C, Mistry M, Roman RJ: Renal effects of glucagon-like peptide in rats. Eur J Pharmacol 2002, 434(3):163-167.

48. van Poppel PC, Netea MG, Smits P, Tack CJ: Vildagliptin improves endotheliumdependent vasodilatation in type 2 diabetes. Diabetes Care 2011, 34(9):2072-2077.

49. Akiyama E, Sugiyama S, Matsuzawa Y, Konishi M, Suzuki H, Nozaki T, Ohba K, Matsubara J, Maeda H, Horibata Y, Sakamoto K, Sugamura K, Yamamuro M, Sumida H, Kaikita K, Iwashita S, Matsui K, Kimura K, Umemura S, Ogawa H: Incremental prognostic significance of peripheral endothelial dysfunction in patients with heart failure with normal left ventricular ejection fraction. J Am Coll Cardiol 2012, 60(18):1778-1786.

doi:10.1186/1475-2840-13-43

Cite this article as: Miyoshi et al:: Effect of vildagliptin, a dipeptidyl peptidase 4 inhibitor, on cardiac hypertrophy induced by chronic beta-adrenergic stimulation in rats. Cardiovascular Diabetology 2014 13:43. 